\title{
Infrared molecular spectra of carbon stars observed by the ISO SWS
}

\author{
W. Aoki, T. Tsuji, K. Ohnaka \\ Institute of Astronomy, The University of Tokyo, Mitaka, Tokyo, Japan
}

\begin{abstract}
.
We carried out observations of bright optical carbon stars with the ISO SWS, and detected the absorption features of $\mathrm{CO}, \mathrm{CS}, \mathrm{CH}, \mathrm{SiS}$ and $\mathrm{HCN}$ in N-type and SC-type stars. The $\mathrm{CH}$ fundamental bands in 3 $4 \mu \mathrm{m}$ in $\mathrm{N}$-type stars are stronger than those in SC-type stars. On the other hand, the first overtone bands of $\mathrm{SiS}$ at $6.6 \mu \mathrm{m}$ were detected in WZ Cas (SC-type), while none of them were detected in N-type stars. These results can be explained well by the lower $\mathrm{C} / \mathrm{O}$ ratio in SC-type stars than in N-type stars. The absorption features of $\operatorname{HCN}\left(\nu_{1}, \nu_{2}+\nu_{3}\right.$ and $\left.\nu_{1}-\nu_{2}\right)$ are stronger in SC-type stars than in N-type stars. This fact can be explained by the cooler atmospheres of our SC-type stars due to the lower $\mathrm{C} / \mathrm{O}$ ratio as well as due to the lower effective temperature, compared with the $\mathrm{N}$-type stars in our sample. The CS bands and the band heads of the $\mathrm{CO}$ fundamental bands are weaker in the observed spectra than in those predicted by our model atmospheres. This result cannot be explained by the present model atmosphere and one possible explanation is the contribution by the emission of CO and CS in the outer atmosphere.
\end{abstract}

\section{Introduction}

Extensive efforts to obtain infrared spectra have been made for carbon stars, because of their importance in the study of the late stages of stellar evolution. However, the wavelength region and the number of objects studied so far are quite limited. The entire spectra from 2.5 to $45 \mu \mathrm{m}$ have become available for the first time by the Short Wavelength Spectrometer (SWS, de Graauw et al. 1996) on board the Infrared Space Observatory (ISO) ${ }^{1}$.

We carried out observations of various types of carbon stars with the ISO SWS, and investigated the vibration-rotation spectra of various molecules in the infrared region which are expected to be quite useful for the understanding of the photosphere and the outer atmosphere of cool evolved stars. In this paper, we report the results of the analysis of molecular absorption features in three $\mathrm{N}$ type and three SC-type stars. SC-type stars are distinguished from N-type stars by the weakness of $\mathrm{C}_{2}$ absorption in the optical region. This fact is generally

\footnotetext{
${ }^{1}$ Based on observations with ISO, an ESA project with instruments funded by ESA Member States (especially the PI countries: France, Germany, the Netherlands and the United Kingdom) with the participation of ISAS and NASA.
} 


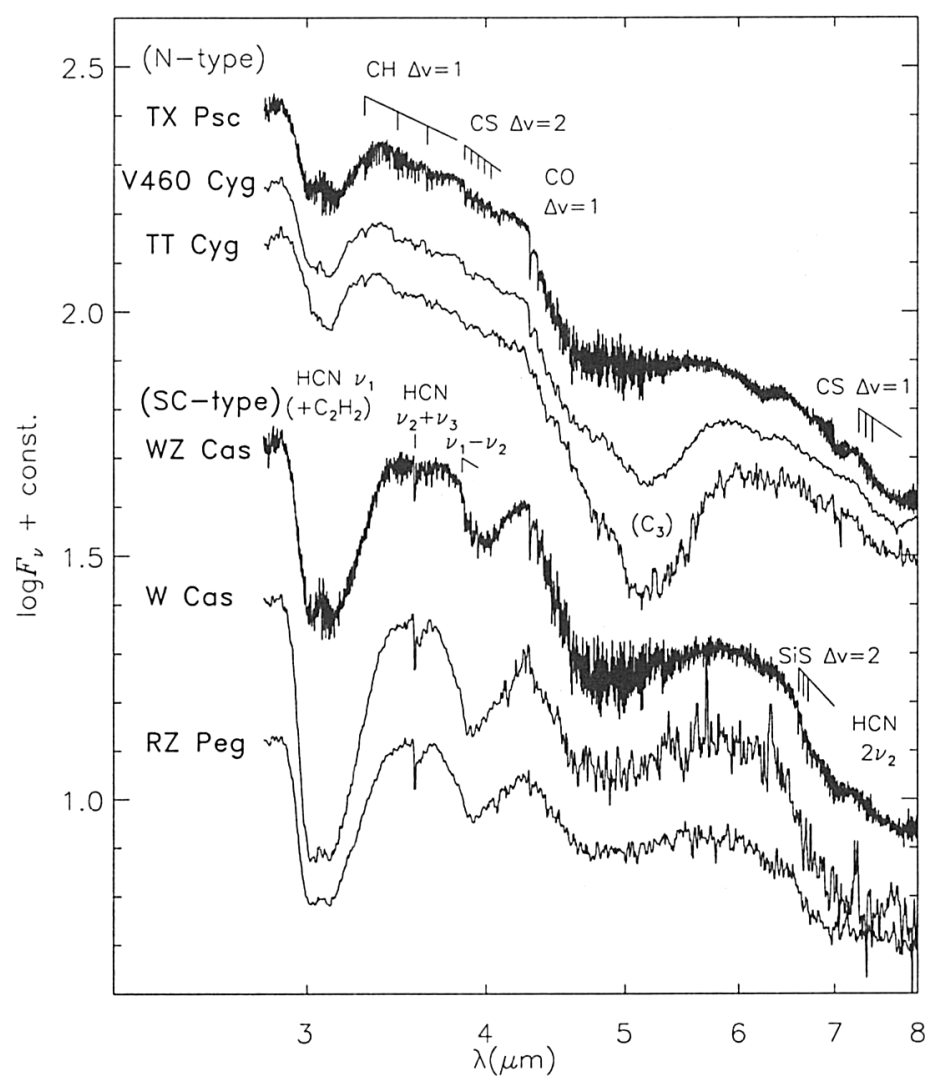

Figure 1. The observed spectra. The upper three are the spectra of $\mathrm{N}$-type stars and the lower three are those of SC-type stars

interpreted that the carbon to oxygen abundance ratios $(\mathrm{C} / \mathrm{O}$ ratios) in $\mathrm{SC}$ type stars are smaller than those in N-type stars. We examine this effect in the molecular features of the infrared region.

\section{Observation, data reduction and analysis}

We observed TX Psc (N-type) and WZ Cas (SC-type) with the SWS06 at a resolution of 1000-2000. We observed other stars with the SWS01 (speed 1) at a resolution of about 200. The details of our observations are given in Table 1. The data reductions were done by the SWS Interactive Analysis software. The discrepancies in flux between different AOT bands were corrected by scaling with respect to the band $1 \mathrm{~B}(2.6-3.0 \mu \mathrm{m})$. The flux discrepancies are at most $10 \%$, except for that at $7 \mu \mathrm{m}$ which is $40 \%$ in the worst case. This correction is not important for the present work on the individual molecular features, though the better flux calibration is necessary for the study of the spectral energy distribution. The spectra from 3 to $8 \mu \mathrm{m}$ of these six carbon stars are shown in Fig. 1. 
In the present work, we compare the observed spectra with synthetic spectra based on our model atmospheres. In the calculation of model atmospheres, the plane parallel geometry, hydrostatic equilibrium and LTE are assumed. The stellar parameters used to specify the model atmospheres are also given in Table 1. The details of the model calculation and the stellar parameters are discussed in Aoki et al. (1998) in which the molecular data used in our analysis are also described. An important point is that the carbon to oxygen abundance ratios (C/O ratios) of SC-type stars are close to unity (e.g. WZ Cas), and hence the backwarming by $\mathrm{CN}$ and $\mathrm{C}_{2}$ is weak in SC-type stars. As a result the atmosphere of SC-type stars is cooler than that of $\mathrm{N}$-type stars of the same effective temperature.

Table 1. Summary of observations and the stellar parameters

\begin{tabular}{lllrccc}
\hline star & spectral type & AOT & obs. date & $T_{\text {eff }}$ & C/O & C/O \\
\hline TX Psc & N-type (C7,2) & SWS06 & 26 Nov 1996 & 3080 & 1.027 & 1.16 \\
V460 Cyg & N-type (C6,3) & SWS01-1 & 11 Jan 1997 & 3230 & 1.062 & \\
TT Cyg & N-type (C5,4) & SWS01-1 & 30 May 1997 & 3050 & - & \\
WZ Cas & SC-type (C9,2JLi) & SWS06 & 22 Jul 1996 & 2980 & 1.010 & \\
W Cas & SC-type (C7,1e) & SWS01-1 & 12 Jan 1997 & 3000 & - & \\
RZ Peg & SC-type (C9,1e) & SWS01-1 & 10 Jun 1996 & 2650 & - & \\
\hline
\end{tabular}

${ }^{1}$ The effective temperature were determined by Ohnaka \& Tsuji (1996). The $T_{\text {eff }}$ of SC-type stars are corrected by Aoki et al. (1998).

${ }^{2} \mathrm{The} \mathrm{C} / \mathrm{O}$ ratio were determined by Lambert et al. (1986).

${ }^{3}$ The $\mathrm{C} / \mathrm{O}$ ratio was determined by Ohnaka (1997).

\section{Molecular features identified in the observed spectra}

We identified the features of several molecules in the observed spectra, especially in the spectra obtained with the SWS06 at the higher resolution. In this section, we discuss the characteristics of molecular features corresponding to the spectral types, and the results of the comparison of the observed features with those predicted by our model atmospheres.

\section{1. $\quad \mathrm{CH}$ and SiS}

As shown in Fig. 1, we identified the $\mathrm{CH}$ fundamental bands in the spectra of three $\mathrm{N}$-type stars. The $\mathrm{CH}$ absorption features in the three SC-type stars are quite weak if any. The weakness of $\mathrm{CH}$ features in SC-type stars can be explained by the small abundance of free carbon atoms which are not locked up into $\mathrm{CO}$ molecules. This is the same characteristic as the weak $\mathrm{C}_{2}$ and $\mathrm{CN}$ absorption in the optical spectra of SC-type stars, which is used as the classification criterion of carbon stars.

We also identified the SiS first overtone bands at $6.6 \mu \mathrm{m}$ in WZ Cas, while none of them is seen in other stars (Fig. 1). In Fig. 2, we show the spectra of TX Psc and WZ Cas with the synthetic spectra of the SiS first overtone bands. The SiS band heads appear in the synthetic spectrum with $\mathrm{C} / \mathrm{O}=1.01$ (i.e. the case of SC-type stars), while no feature appears in the synthetic spectrum with 


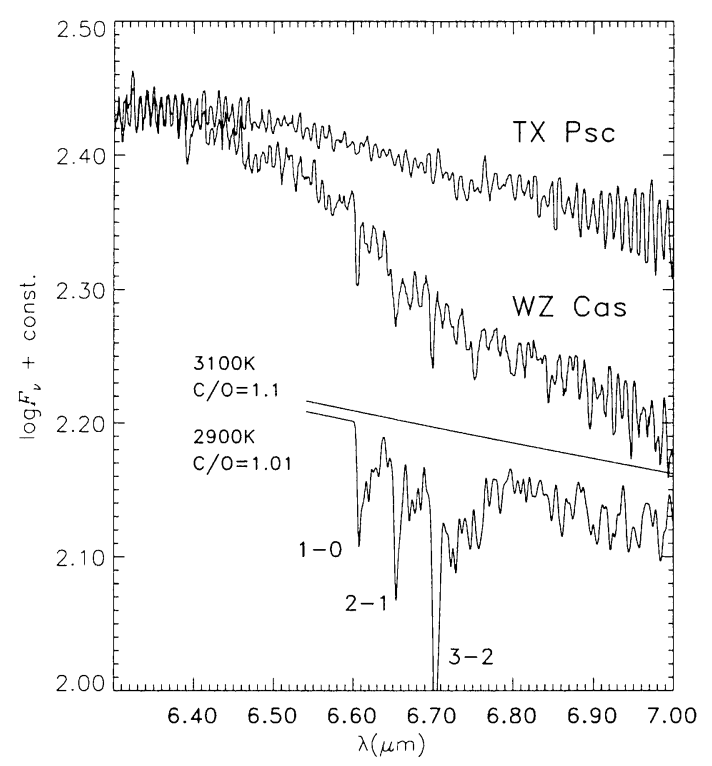

Figure 2. The 6-7 $\mu \mathrm{m}$ region of the observed spectra of TX Psc and WZ Cas, and the synthetic spectra of the SiS first overtone bands. The broad absorption in the wavelength region longer than $6.5 \mu \mathrm{m}$ of the spectrum of WZ Cas is due to the $2 \nu_{2}$ bands of $\mathrm{HCN}$

$\mathrm{C} / \mathrm{O}=1.1$ (i.e. the cases of $\mathrm{N}$-type stars). The strong dependence of the SiS absorption on the $\mathrm{C} / \mathrm{O}$ ratio is qualitatively explained as follows; in carbon rich case as $\mathrm{N}$-type stars (e.g. $\mathrm{C} / \mathrm{O} \geq 1.02$ ), carbon is abundant enough to consume almost all sulfur atoms by forming CS molecules. On the other hand, in SC-type stars $(\mathrm{C} / \mathrm{O} \sim 1.01)$, the abundance of carbon which is not locked up into $\mathrm{CO}$ molecules is too low to consume sulfur atoms by forming CS molecules, and the remaining sulfur can form SiS. The coolness of the atmospheres of SC-type stars also promotes the formation of SiS.

\subsection{HCN vibration-rotation spectra}

As shown in Fig. 1, we detected the well known $3 \mu \mathrm{m}$ band which is attributed to $\mathrm{HCN}$ and/or $\mathrm{C}_{2} \mathrm{H}_{2}$. We also detected the $\mathrm{HCN} \nu_{2}+\nu_{3}$ bands at $3.56 \mu \mathrm{m}$ in the three SC-type stars. The $\nu_{1}-\nu_{2}$ bands at $3.9 \mu \mathrm{m}$ would appear in the spectrum of WZ Cas, though these features blend with the CS first overtone bands.

In Fig. 3, we show the synthetic spectra of the above three bands of HCN together with the observed ones for TX Psc and WZ Cas. The vibration-rotation spectra of $\mathrm{H}^{13} \mathrm{CN}$ and of $\mathrm{C}_{2} \mathrm{H}_{2}$ are not included in the calculation of the $3 \mu \mathrm{m}$ band, although the absorption of $\mathrm{H}^{13} \mathrm{CN}$ may be important in WZ Cas because of the lower ${ }^{12} \mathrm{C} /{ }^{13} \mathrm{C}$ ratio (see Table 1 ).

One important result is that the absorption of HCN in our SC-type stars is stronger than that in the $\mathrm{N}$-type stars (see Fig. 1), in contrast with the weakness of absorption features of other carbon-bearing molecules (e.g. $\mathrm{C}_{2}, \mathrm{CN}$ and $\mathrm{CH}$ ) in $\mathrm{SC}$-type stars because of the lower $\mathrm{C} / \mathrm{O}$ ratio. This is because the absorption 


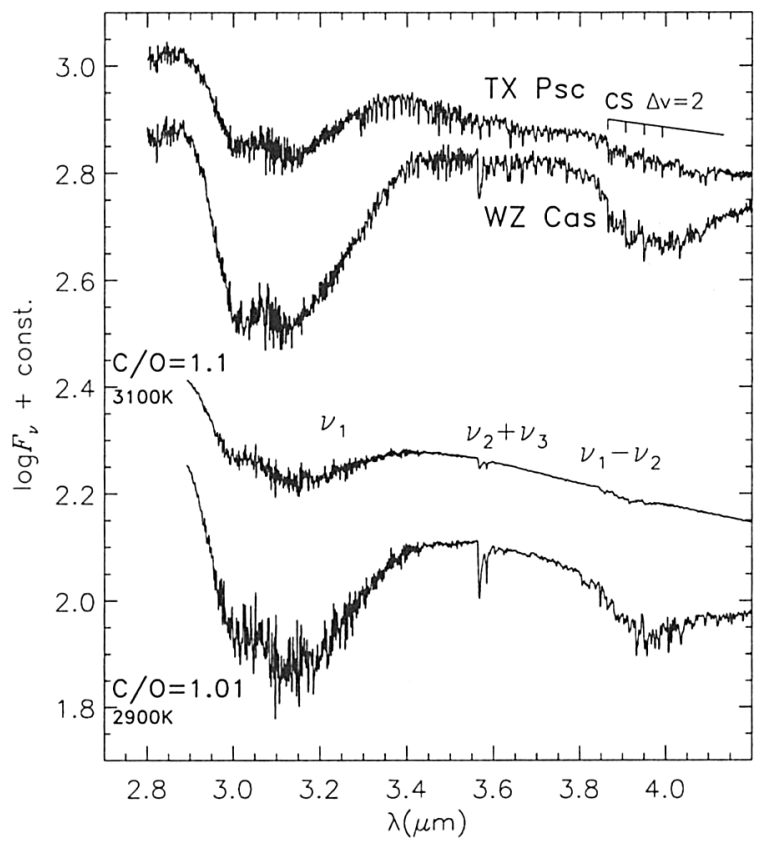

Figure 3. The observed spectra of TX Psc and WZ Cas, and the synthetic spectra of the vibration-rotation bands of $\mathrm{HCN}$

of $\mathrm{HCN}$ is very sensitive to the temperature structure of the atmosphere. Since the atmospheres of SC-type stars are cooler than those of N-type stars with the same effective temperature, the HCN absorption in SC-type stars could be stronger than that of $\mathrm{N}$-type stars, in spite of the lower $\mathrm{C} / \mathrm{O}$ ratio. (However, we cannot conclude that the HCN absorption features in SC-type stars are generally stronger than those in N-type stars, because the HCN absorption is also sensitive to the effective temperature.)

\section{3. $C O$ and $\mathrm{CS}$}

The CS first overtone and CO fundamental bands are clearly identified (Fig. 1). However, the absorption in the observed spectra is weaker than in the spectra predicted by our model atmospheres as shown in Fig. 4. The fundamental bands of CS are seen at $7 \mu \mathrm{m}$ in the spectra of TX Psc (Fig. 1), but they are very weak. Since these molecules are quite stable in the atmospheres of cool carbon stars, it is difficult to explain these facts by the small changes of the temperature structure of the model atmosphere or of the stellar parameters.

One possible reason for the weakness of these absorption features is the contribution of the emission of $\mathrm{CO}$ and $\mathrm{CS}$ in the outer atmosphere. Our observations of oxygen-rich giants with the ISO SWS (Tsuji et al. 1997) revealed that the absorption features of $\mathrm{CO}$ and $\mathrm{SiO}$ in $\mathrm{M}$ giants are weaker, while the absorption of $\mathrm{H}_{2} \mathrm{O}$ and $\mathrm{CO}_{2}$ are stronger, than those predicted by our model atmosphere, and we proposed the existence of warm molecular envelopes around the photospheres. We suggest now that warm molecular envelopes exist not only in oxygen-rich giants but also in carbon stars. 


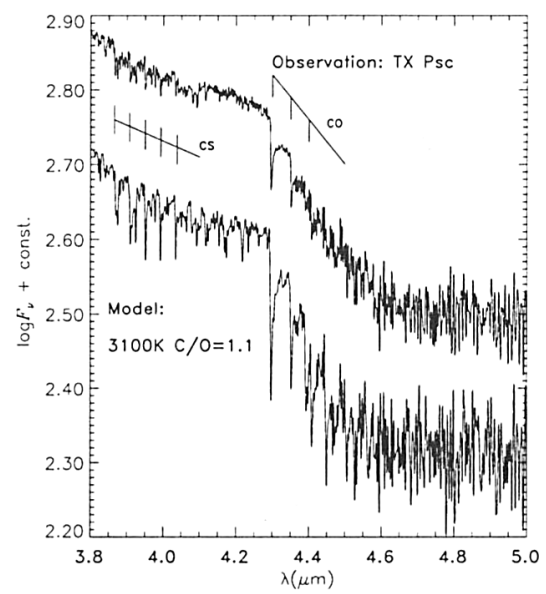

Figure 4. The observed spectrum of TX Psc and the synthetic spectrum of the CS first overtone and the CO fundamental bands

\section{Discussion and concluding remarks}

In addition to the features identified in the previous section, we identified HCN $2 \nu_{2}$ bands at around $7 \mu \mathrm{m}$ in SC-type stars, and $\mathrm{C}_{3}$ at $5 \mu \mathrm{m}$ in TT Cyg and V460 Cyg (see Fig. 1). $\mathrm{C}_{2} \mathrm{H}_{2}$ may contribute to the absorption at around 3 and $7 \mu \mathrm{m}$, but the contribution is not large in our sample. At the present stage, most of the absorption features in this wavelength region are identified, and the dependence on the spectral type is explained well for N-type and SC-type stars. The importance of the $\mathrm{C} / \mathrm{O}$ ratio for the structures of atmospheres as well as for the molecular formation is shown by the analyses of the molecular features.

However, the strength of the absorption of $\mathrm{CO}$ and CS can not be explained well by our model atmospheres. For detailed and quantitative discussion on the molecular spectra of carbon stars, we need more realistic model atmospheres and more precise parameters of atmospheres, and may need to take the time variation of the spectra into account. The detailed models of the outer atmosphere are also required to study the implications of these features.

Acknowledgments. We are grateful to Prof. H. Okuda for taking the initiative of our ISO project. We would like to thank Dr. I. Yamamura for helpful support in the data reduction, and also thank the SWS Instrument Dedicated Team for making available the SWS Interactive Analysis software. W. A. and K.O. are supported by JSPS Fellowship for young scientists.

\section{References}

Aoki W., Tsuji T., Ohnaka K., 1998, A\&A 340, 222

de Graauw Th., et al., 1996, A\&A 315, L49

Lambert D.L., Gustafsson B., Eriksson K., Hinkle K.H., 1986, ApJS 62, 373

Ohnaka K., 1997, Ph. D. Thesis, The University of Tokyo

Ohnaka K., Tsuji T., 1996, A\&A 310, 933

Tsuji T., Ohnaka K., Aoki W., Yamamura I., 1997, A\&A 320, L1 